\title{
Estimación del comportamiento productivo y parámetros genéticos de características predestete en bovinos de carne (Bos taurus) y sus cruzas, VIII Región, Chile
}

\author{
Estimation of pre-weaning productive performance and genetic parameters in beef cattle \\ (Bos taurus) and crosses, VIII Region of Chile
}

\author{
E G Cienfuegos-Rivas ${ }^{3}$, M A R de Orúe-Ríos ${ }^{2}$, M Briones-Luengo ${ }^{1}$, J C Martínez-González ${ }^{3}$ \\ ${ }^{1}$ Universidad de Concepción, Facultad de Medicina Veterinaria, Departamento de Ciencias Pecuarias, Chillán, Chile. \\ 2 Tesista de la Universidad de Concepción, Facultad de Medicina Veterinaria, Chillán, Chile. \\ ${ }^{3}$ Universidad Autónoma de Tamaulipas, Unidad Académica Multidisciplinaria Agronomía y Ciencias, División de Estudios de \\ Postgrado e Investigación, Cd. Victoria, Tamaulipas, México.
}

\begin{abstract}
SUMMARY
The objective was to determine the productive performance in body weight change from birth until weaning, of calves sired by Angus, Hereford and Polled Hereford with Clavel, Hereford, Holstein, Polled Hereford, and Hybrid (different Holstein and Hereford combinations) cows in a beef cattle herd in the dry area of the Nuble, province of the VIII Region in Chile. The sires and cows were classified according to their phenotypic characteristics. The productive performance was evaluated by analyzing 1,119 production records from year 1990 to 2000 for the variables: birth weight (PN, N=1,119), adjusted weaning weight (PD205, N=660) and preweaning daily gain (GDP; $\mathrm{N}=660$ ) using the least squares method to determine the effects of breed group of the sire (GRT), breed group of the dam (GRV), and contemporary groups (GC), with age of dam (EDV) as a covariable, on PN, PD205 and GDP. Genetic and environmental parameters were estimated using an animal model with MTDFREML methodology, through univariable and bivariable analyses to estimate variance and covariance components. GRT and the GC had a significant effect $(\mathrm{P}<0.05)$ on $\mathrm{PN}$ and PD205 but did not affect GDP (P•0.05). Calves sired by Hereford bulls were 7.5, 12.5 y $14.0 \%$ above the herd average for PN, PD205 and GDP, respectively. The GRV did not have an effect $(\mathrm{P} \bullet 0.05)$ on PN, PD205 and GDP. Heritabilities $\left(\mathrm{h}^{2}\right)$ calculated were 0.49 for PN, 0.15 for PD205 and 0.35 for GDP. Genetic correlation between PN and PD205 was 0.23 . Within the GRT the $\mathrm{h}^{2}$ for PN and PD205 were 0.69 and 0.21 for Angus and 0.47 and 0.23 for Polled Hereford, respectively.
\end{abstract}

Palabras clave: terneros, ganancia de peso, herencia.

Key words: calves, pre-weaning traits, heritability.

\section{INTRODUCCION}

En la gran mayoría de las explotaciones ganaderas productoras de carne, las características que mayor influencia tienen en los costos de operación son: la eficiencia reproductiva de la vaca, que se mide según el porcentaje de terneros destetados y el comportamiento productivo del ternero (Dickerson 1978). En cuanto a este último, el peso de los terneros al destete es uno de los componentes del comportamiento productivo que inciden en el retorno neto de la explotación, debido a que su maximización está asociada al incremento en el volumen de producción, el tamaño corporal (maduro), la producción lechera de la madre y la tasa de crecimiento en la progenie (Magofke 1991) y al estrecho equilibrio que

Aceptado: 23.08.2005

* Fono-Fax: 041-238930. EM: mdeoruee@udec.cl. Correo: Casilla 2451, Concepción. existe entre la demanda de nutrientes y la disponibilidad de los recursos del predio.

Por otro lado, el mejoramiento de la producción se puede lograr mediante la introducción, por cruzas, de nuevos genotipos que, en ciertas ocasiones, pueden conducir a un incremento en la presentación de los partos distócicos provocados por un mayor tamaño fetal (Magofke 1991) que termina por afectar gravemente la rentabilidad del sistema productivo (Varona y col 1999).

Las características de porcentaje de partos distócicos, peso al nacimiento, supervivencia perinatal, peso al destete y porcentaje de terneros destetados, son afectadas por factores genéticos, como la raza de los progenitores y el grado de heterosis que presente la cría (Alejo y col 2000, Martínez 1986), y por factores ambientales, como el manejo del predio, la nutrición animal, la edad de la vaca, su condición corporal durante la gestación y el postparto, su amplitud pélvica y el sexo de la cría (Simm 1998, Martínez 1986). 
El reto para los ganaderos de los países latinoamericanos es, entonces, lograr la identificación de aquellos animales que logran obtener un máximo desempeño productivo en un determinado ambiente agroecológico. Asimismo, multiplicar y perpetuar su genotipo por medio de cruzamientos, práctica que hoy en día es favorecida para elevar la productividad, la rentabilidad, la eficiencia y la sustentación de los sistemas productivos de carne (Martínez-González y col 2003). Situación que fácilmente se puede adaptar a las condiciones de los sistemas de producción de carne de Chile, considerando que cada combinación de genes se expresa diferente según el ambiente en el cual se han desarrollado.

Por otro lado, las características productivas (de crecimiento), antes mencionadas, presentan índices de herencia $\left(\mathrm{h}^{2}\right)$ de valores medios a altos, lo que sugiere que se pueden establecer programas de mejoramiento genético en poblaciones de bovinos de carne. Ejemplo de lo anterior, lo documentan Bullock y col (1993), que a partir de 572.446 datos de la American Polled Hereford Association (APHA) encontraron un $\mathrm{h}^{2}$ para los pesos al nacimiento y al destete de 0,49 y 0,24 , respectivamente. Por su parte, Tosh y col (1999) al estudiar 5.880 registros productivos de vacas $\mathrm{F}_{1}$ (Hereford, Angus o Shorthorn con Charoláis, Simmental o Limousine) y toros híbridos (Hereford x Angus) de dos estaciones de investigación en Canadá, tomados entre 1982 y 1994, reportaron un $\mathrm{h}^{2}$ de 0,51 y 0,33 para los pesos al nacimiento y al destete, respectivamente. Similarmente, Elzo y Wakeman (1998) luego de analizar 1.581 registros de cruzas de distinta proporción racial entre Angus y Brahman, reportaron un $\mathrm{h}^{2}$ que varió entre 0,13 a 0,22 y de 0,15 a 0,25 para los pesos al nacimiento y al destete, respectivamente, y correlaciones genéticas $\left(\mathrm{r}_{\mathrm{G}}\right)$ entre ambos pesos desde 0,24 a 0,26.

Los objetivos de esta investigación fueron: evaluar el comportamiento productivo y la estimación de componentes de varianza genética y ambiental de terneros obtenidos entre las cruzas de toros Angus, Hereford y Polled Hereford y vacas Clavel, Hereford, Holstein, Polled Hereford e Híbridas (cruzas Holstein x Hereford), en un predio de la comuna de Ninhue, provincia de Nuble, VIII Región de Chile, para las características del peso al nacimiento, peso al destete y ganancia de peso diaria predestete.

\section{MATERIAL Y METODOS}

Descripción geográfica. Los datos fueron obtenidos de los registros productivos realizados entre los años 1990 y 2000 en un predio de 400 hectáreas de extensión que se encuentra ubicado en la comuna de Ninhue, provincia de Ñuble, VIII Región de Chile. En este predio predominan las praderas naturales típicas del secano interior (Ruiz 1996) y, geográficamente, está ubicado dentro de la zona agroecológica al oriente de la cordillera de la costa, en- tre los ríos Mataquito (Curicó) e Itata, entre los paralelos $35^{\circ}$ latitud norte y $36^{\circ} 40^{\prime}$ longitud oeste. El clima es del tipo mediterráneo subhúmedo, caracterizado en invierno por períodos fríos y húmedos con escasas heladas y en verano por períodos secos y calurosos (Del Pozo y Del Canto 1999).

Grupos raciales y manejo. La masa ganadera original de este predio estaba constituida por animales de las razas Holstein, Frisón rojo y negro. Luego de un programa de absorción racial para convertir el predio en productor cárnico, se observaron las siguientes razas al momento del estudio: Clavel (de gran habilidad cárnica), Hereford, Polled Hereford, Holstein e Híbridas (cruzas Holstein x Hereford) como grupo racial de la vaca (GRV) y Angus, Limousine, Simmental, Piedmontese, Hereford y Polled Hereford como grupo racial del toro (GRT). El manejo predial consistía en pariciones de primavera con encaste entre los meses de octubre y noviembre, utilizando inseminación natural y artificial. La alimentación de los animales consistía en pastoreo de praderas naturales mejoradas y suplemento invernal especialmente en vacas.

Edición de los datos. De los 1.368 registros de la base original, el 18,2\% no fueron considerados en el estudio. Dentro de las principales razones están: el bajo número de crías para algún GRT, registros repetidos o incongruentes, falta de datos (fecha de nacimiento o el peso al nacimiento (PN) y al destete (PD) en forma conjunta), y aquellos PD fuera del rango 150-250 días. De esta forma, el estudio se basó en 1.119 registros productivos, todos con PN y 660 con PD.

El archivo genealógico de la masa ganadera lo formaban 1.373 animales (25 progenitores machos y 229 progenitoras hembras). Dentro del GRT, la cantidad de registros productivos y el número total de animales en la genealogía se observan en el cuadro 1, para el GRV el número de registros fueron Clavel $(\mathrm{N}=62)$, Hereford $(\mathrm{N}=233)$, Polled Hereford $(\mathrm{N}=287)$, Holstein $(\mathrm{N}=80)$ e Híbridas $(\mathrm{N}=457)$.

El peso al destete se ajustó a los 205 días (PD205), de igual forma que lo realizado por Kaps y col (1999). Se calculó una nueva variable, ganancia de peso diaria predestete (GDP), restando el PN al PD y dividiendo el resultado por los días al destete. Similarmente, con las medias de mínimos cuadrados se realizó un ajuste por sexo del ternero a las variables PN y PD205, el cual consistió en obtener un factor, dividiendo el PN medio de machos por el PN medio de hembras, para luego multiplicarlo por los pesos de las hembras (Spide y col 1981).

Modelo estadístico general. Con base en los registros productivos se consideraron los siguientes supuestos para el planteamiento del modelo estadístico general: el GRT son animales Angus y Polled Hereford puros, y el GRV son animales Clavel, Hereford, Polled Hereford, Holstein 
Cuadro 1. Número de registros $(\mathrm{N})$ y número total de animales en genealogía para la masa ganadera en estudio y según grupo racial del toro (GRT).

Number of records number $(\mathrm{N})$ and total number of animals in genealogy for the cattle studied, according to breed group of the sire (GRT)

\begin{tabular}{|c|c|c|}
\hline & $\mathrm{N}$ & $\begin{array}{l}\text { Total de animales } \\
\text { en genealogía }\end{array}$ \\
\hline $\begin{array}{l}\text { Masa ganadera } \\
\text { GRT }\end{array}$ & 1.119 & $1.373^{*}$ \\
\hline$* *$ Angus & 400 & 583 \\
\hline$* *$ Hereford & 167 & 247 \\
\hline **Polled Hereford & 552 & 711 \\
\hline \multicolumn{3}{|c|}{$\begin{array}{l}\text { * Número total de individuos en genealogía (toro, padre del toro y } \\
\text { abuelo materno del toro). }\end{array}$} \\
\hline misma vaca pued & $\begin{array}{l}\text { es en gen } \\
\text { la misma } \\
\text { de mas } \\
\text { cruzado } \\
168 \text { oce }\end{array}$ & $\begin{array}{l}\text { alogía de Angus, Hereford y } \\
\text { cantidad que el número total } \\
\text { ganadera debido a que una } \\
\text { con toros Hereford y Polled } \\
\text { iones. }\end{array}$ \\
\hline
\end{tabular}

puros e Híbridas, los cuales se consideraron como genotipo debido a que no se contó con la información de las proporciones genéticas con que participaban las distintas razas que la conformaron, por lo tanto se tomó en consideración al GRT y GRV en el modelo animal utilizado para PN, PD205 y GDP con el fin de remover el efecto multirracial.

El modelo en notación matricial fue: $\mathrm{Y}=\mathrm{Xb}+\mathrm{Wg}+\mathrm{Zu}+\mathrm{e}$ donde:

$\mathrm{Y}=$ Vector de observaciones; $\mathrm{X}=$ matriz de incidencia para los efectos fijos (o sea, $\mathrm{X}=[\mathrm{V} \mathrm{T} \mathrm{E}]$, donde $\mathrm{V}=$ matriz de incidencia del GRV, $\mathrm{T}=$ matriz de incidencia del GRT y E = matriz de incidencia para la covariable edad de la vaca (EDV)); $b=$ vector de los efectos fijos desconocidos para el GRV, GRT y EDV; W = matriz de incidencia para el efecto año-estación de grupos contemporáneos $(\mathrm{GC}) ; \mathrm{g}=$ vector de efectos fijos desconocidos del GC; $Z$ = matriz de incidencia que relaciona los registros con los animales; $\mathrm{u}=$ vector de los efectos aleatorios de los animales; y e $=$ vector de los efectos residuales.

Modelo estadístico dentro de GRT. Para este modelo se consideró al grupo racial de la cría (GRC) como un genotipo único, debido a que no se contó con las proporciones genéticas maternas. El modelo animal utilizado para PN, PD205 y GDP incluyó sólo dos de las tres razas paternas (Angus y Polled Hereford) debido a que en la raza Hereford se tenían 167 registros productivos de un solo toro distribuido en los años de estudio.

El modelo en notación matricial fue: $\mathrm{Y}=\mathrm{Xb}+\mathrm{Zu}+\mathrm{e}$ donde:

$\mathrm{Y}=$ vector de observaciones; $\mathrm{X}=$ matriz de incidencia para los efectos fijos (donde, $\mathrm{X}=[\mathrm{H} \mathrm{C} \mathrm{E}]$; con $\mathrm{H}=$ ma- triz de incidencia para el GC; $\mathrm{C}=$ matriz de incidencia del GRC; y $\mathrm{E}=$ matriz de incidencia para la covariable EDV); $b=$ vector de los efectos fijos desconocidos para $\mathrm{GC}$, GRC y EDV; Z = matriz de incidencia que relaciona los registros con los animales; $\mathrm{u}=$ vector de los efectos aleatorios de los animales; $\mathrm{y}$ e = vector de los efectos residuales.

La distribución conjunta de $\mathrm{u}$ y e fue bivariada con

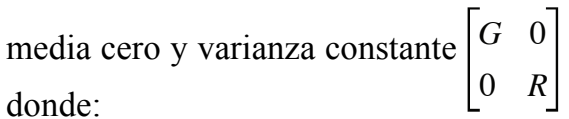

G y R son las matrices de varianzas y covarianzas, genéticas y residuales, respectivamente. Para obtener los componentes de varianza de la matriz $\mathrm{G}$, ésta se consideró como $G=G_{0}$ A, donde $G_{0}$ es la matriz de varianzas y covarianzas dentro del efecto animal para las variables consideradas, A es el numerador de la matriz de parentesco entre los animales y es el producto Kronecker. El coeficiente de consanguinidad obtenido fue de 0,24.

Estimación de componentes de varianza. Los componentes de varianza y covarianza, genético y ambiental fueron estimados con análisis bivariados (dos características al mismo tiempo), usando el método de máxima verosimilitud restringida libre de derivadas para múltiples variables (MTDFREML, siglas del inglés Multiple Trait Derivative Free Restricted Maximum Likelihood), sugerido por Boldman y col (1995). Los valores iniciales de los análisis bivariados fueron las soluciones de los análisis univariados para cada característica considerada. La convergencia se alcanzó cuando los valores de -2 Log likelihood fue $<10^{-8}$. El máximo global se alcanzó cuando tres reinicios produjeron convergencias sin cambios en los primeros tres decimales del valor $\mathrm{F}$.

Estimación de parámetros genéticos. $\mathrm{El} \mathrm{h}^{2} \mathrm{y}$ las correlaciones genética $\left(\mathrm{r}_{\mathrm{G}}\right)$ y fenotípica $\left(\mathrm{r}_{\mathrm{F}}\right)$ fueron estimados usando el método MTDFREML, el error estándar para $\mathrm{h}^{2}$ fue aproximado con el método de Swiger y col (1964).

Evaluación de comportamiento productivo. Para evaluar el comportamiento productivo de los animales se utilizó el modelo general y el modelo dentro de GRT descrito anteriormente, obteniéndose las medias de mínimos cuadrados (Hatcher y Stepanski 1997) para PN, PD205 y GDP y los predictores para los efectos fijos descritos en el modelo.

\section{RESULTADOS Y DISCUSION}

Medias fenotípicas generales. Las medias fenotípicas de las variables PN, PD205 y GDP para toda la ganadería fueron: $37,7 \pm 6,1 \mathrm{~kg}, 210,5 \pm 41,4 \mathrm{~kg}$ y $0,884 \pm 0,191 \mathrm{~kg}$, respectivamente. Dentro de GRT la raza Angus tuvo los valores para peso al destete más bajos $(221,6 \mathrm{~kg}$, cuadro 4$)$. Sin embargo, mostró una mejor habilidad combinatoria 
con el GRV Holstein (257,3 kg, cuadro 2). Los valores encontrados para el GRT Angus son similares a los descritos por Wilson y col (1986); Winder y col (1990); Bullock y col (1993) y Tosh y col (1999). En otras investigaciones se reportan rangos desde los $172 \mathrm{~kg}$ (Veseth y col 1993) hasta los 260,3 kg (Kaps y col 1999) para peso al destete ajustado a 205 días.

Efecto de sexo de la cría. Para la variable PN el sexo de la cría tuvo un efecto significativo $(\mathrm{P}<0,001)$, siendo los machos cerca de $3 \mathrm{~kg}$ más pesados que las hembras (cuadro 3), lo cual coincide con lo reportado por Martínez y col (1998). La diferencia entre machos y hembras $(61,4$ $\mathrm{kg}$, cuadro 3) se hizo más evidente en PD205 ( $\mathrm{P}=0,03)$, valor por sobre a lo encontrado por Kaps y col (1999), quienes para la raza Angus reportan diferencias entre machos y hembras desde 30 hasta $50 \mathrm{~kg}$. En la variable GDP, no hubo diferencia significativa para el sexo de la cría. Es importante recalcar que debido a que las diferencias entre machos y hembras dentro del grupo racial de los progenitores han sido ampliamente documentadas para las variables productivas predestete se decidió ajustar por sexo de la cría para las variables PN y PD205.

Efecto de grupo contemporáneo. Para las variables PN y $\mathrm{PD} 205$ se encontraron diferencias $(\mathrm{P}<0,001)$ entre los 21 grupos contemporáneos evaluados. En el caso de los grupos contemporáneos, existen reportes muy variados de-

Cuadro 2. Resumen del total de observaciones para peso al nacimiento (PN), peso al destete (PD205) y ganancia de peso predestete (GPD) de la masa ganadera y según grupo racial de toros y vacas.

Summary of the observations for birth weight (PN), adjusted weaning weight (PD205) and pre-weaning daily gain (GPD) of the cattle, according to breed groups of sires and dam.

\begin{tabular}{|c|c|c|c|c|c|c|}
\hline \multirow{3}{*}{$\begin{array}{l}\text { Grupos } \\
\text { Racial de } \\
\text { la vaca }\end{array}$} & & \multicolumn{5}{|c|}{ Racial del toro } \\
\hline & & \multicolumn{2}{|r|}{ Angus } & Hereford & \multicolumn{2}{|c|}{ Hereford } \\
\hline & & PN & PD205 GPD & PN PD205 GPD & PN PD205 & GPD \\
\hline \multirow[t]{3}{*}{ Clavel } & $\mathrm{N}$ & 24 & $14 \quad 14$ & & 38 & 14 \\
\hline & $\bar{X}$ & 30,7 & $235,8 \quad 0,86$ & & $45,1270,8$ & 1,02 \\
\hline & $\mathrm{DE}$ & 5,9 & $48,5 \quad 0,20$ & & $8,9 \quad 45,8$ & 0,14 \\
\hline \multirow[t]{3}{*}{ Hereford } & $\mathrm{N}$ & 61 & $42 \quad 42$ & $\begin{array}{lll}32 & 21 & 21\end{array}$ & $140 \quad 61$ & 61 \\
\hline & $\bar{X}$ & 38,5 & $236,0 \quad 0,83$ & $42,4 \quad 271,2 \quad 0,98$ & $41,6236,7$ & 0,86 \\
\hline & $\mathrm{DE}$ & 6,0 & $49,4 \quad 0,16$ & $4,4 \quad 0,17$ & $6,6 \quad 50,2$ & 0,18 \\
\hline \multirow[t]{3}{*}{ Híbrida } & $\mathrm{N}$ & 207 & $140 \quad 140$ & $47 \quad 47$ & $192 \quad 116$ & 116 \\
\hline & $\bar{X}$ & 36,5 & $226,0 \quad 0,77$ & $41,7 \quad 263,4 \quad 0,93$ & $37,8239,7$ & 0,82 \\
\hline & $\mathrm{DE}$ & 5,6 & $\begin{array}{lll}53,7 & 0,18\end{array}$ & $\begin{array}{llll}5,5 & 65,8 & 0,20\end{array}$ & $4,8 \quad 58,6$ & 0,16 \\
\hline \multirow[t]{3}{*}{ Holstein } & $\mathrm{N}$ & 36 & $\begin{array}{ll}18 & 18\end{array}$ & $\begin{array}{lll}10 & 8 & 8\end{array}$ & $34 \quad 11$ & 11 \\
\hline & $\bar{X}$ & 40,6 & $257,3 \quad 0,94$ & $\begin{array}{llll}39,0 & 280,7 & 0,98\end{array}$ & $42,5 \quad 242,0$ & 0,91 \\
\hline & $\mathrm{DE}$ & 5,5 & $24,9 \quad 0,18$ & $\begin{array}{lll}5,8 & 64,4 & 0,15\end{array}$ & $6,4 \quad 46,9$ & 0,20 \\
\hline Polled & $\mathrm{N}$ & 72 & $\begin{array}{ll}46 & 46\end{array}$ & $\begin{array}{lll}67 & 51 & 51\end{array}$ & $148 \quad 70$ & 70 \\
\hline \multirow[t]{2}{*}{ Hereford } & $\bar{X}$ & 36,3 & $223,0 \quad 0,76$ & $41,6 \quad 267,90,91$ & $40,1246,0$ & 0,85 \\
\hline & $\mathrm{DE}$ & 5,1 & $\begin{array}{ll}49,5 & 0,17\end{array}$ & $\begin{array}{llll}5,5 & 55,1 & 0,14\end{array}$ & $5,0 \quad 69,0$ & 0,19 \\
\hline
\end{tabular}

N=Número de observaciones. $\bar{X}=$ Media del peso en $\mathrm{kg}$. $\mathrm{DE}=$ Desviación estándar en $\mathrm{kg}$. bidos principalmente a la definición misma de grupo contemporáneo. En este estudio, la población inicial presentó mayores pesos al nacimiento y al destete (figura 1), debido posiblemente a que a partir de 1989 se inició un

Cuadro 3. Medias de mínimos cuadrados para peso al nacimiento (PN), peso al destete ajustado a 205 días (PD205) y ganancia de peso diaria al destete (GDP), ajustado para sexo de la cría y edad de la vaca.

Least squares means for birth weight (PN), adjusted weaning weight (PD205) and pre-weaning daily gain (GDP) adjusted for sex of calf and age of dam.

\begin{tabular}{|c|c|c|c|c|c|c|c|c|c|}
\hline \multirow[b]{2}{*}{ Sexo cría } & \multicolumn{3}{|c|}{ PN (kg) } & \multicolumn{3}{|c|}{ PD205 $(\mathrm{kg})$} & \multicolumn{3}{|c|}{ GDP (kg) } \\
\hline & $\mathrm{N}$ & $\bar{X}$ & $\overline{\mathrm{EE}}$ & & $\bar{X}$ & $\overline{\mathrm{EE}}$ & $\mathrm{N}$ & $\bar{X}$ & $\overline{\mathrm{EE}}$ \\
\hline Macho & 561 & $40,4^{\mathrm{a}}$ & 0,41 & 367 & $276,3^{\mathrm{a}}$ & 4,4 & 367 & $0,86^{\mathrm{a}}$ & 0,19 \\
\hline Hembra & 558 & $37,4^{\mathrm{b}}$ & 0,40 & 293 & $214,9^{\mathrm{b}}$ & 4,4 & 293 & $0,83^{\mathrm{a}}$ & 0,21 \\
\hline
\end{tabular}

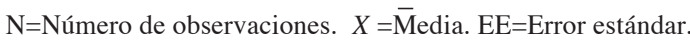

a, b son diferentes estadísticamente $(\mathrm{P}<0,05)$.
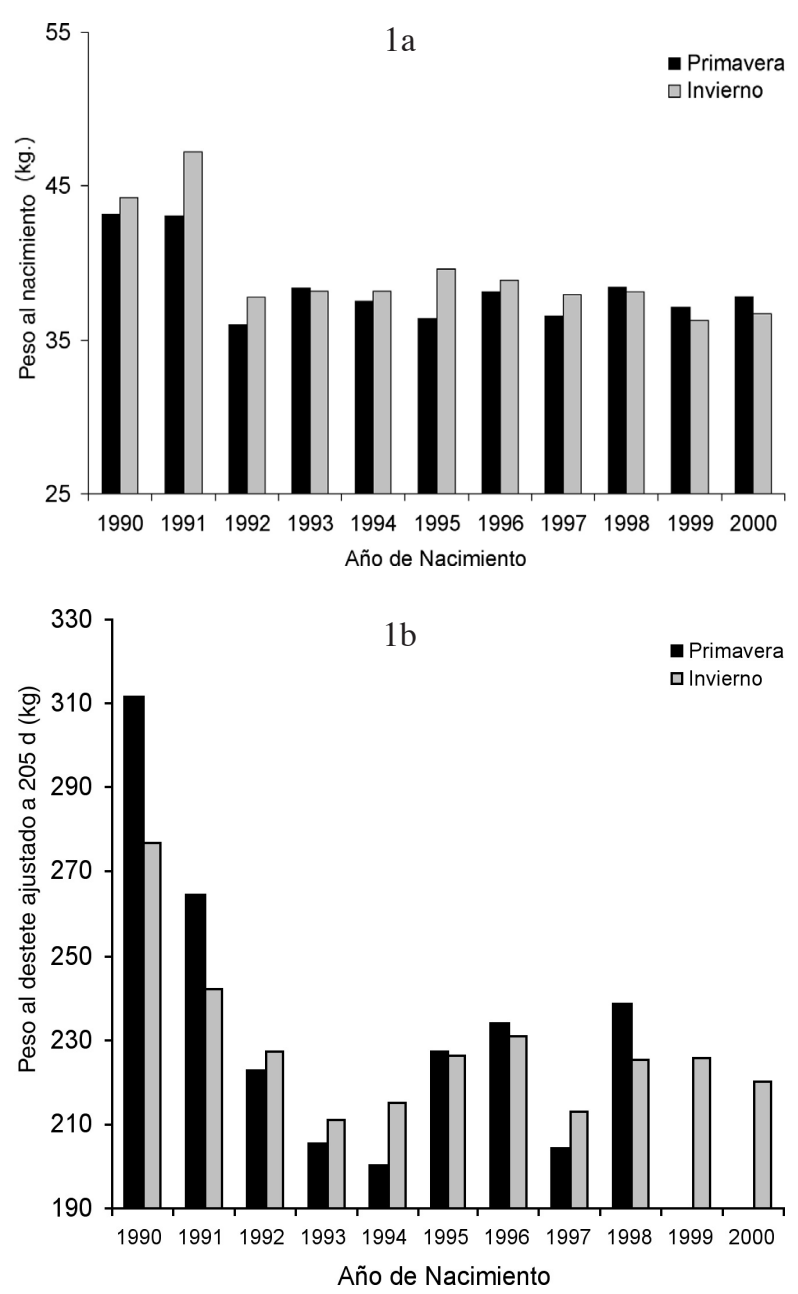

Figura 1. Peso al nacimiento (1a) y peso al destete ajustado a 205 días (1b), por grupo contemporáneo.

Birth weight (1a) and adjusted weaning weight (1b) of animals born each year. 
programa de absorción de la raza Hereford en el predio con la introducción de toros probados, principalmente de la raza Angus. Este cambio en el manejo del hato produjo una reducción en PN y PD205 $(\mathrm{P}<0,01)$ de los terneros nacidos entre 1990 a 1993.

El efecto de año de nacimiento sobre PN a partir del año 1993 se mantuvo sin cambios significativos $(\mathrm{P}>0,05)$ tanto en primavera como en invierno. El año de nacimiento sobre PD205 tuvo un comportamiento errático $(\mathrm{P}<0,01)$ en las dos estaciones evaluadas. En lo que se refiere a año de nacimiento, Martínez y col (1998), en un estudio con crías de diferentes cruzas de Bos taurus y Bos indicus, no encontraron diferencias significativas $(\mathrm{P} \geq 0,05)$ para el $\mathrm{PN}$, mientras que Lunstra y Cundiff (2003) hallaron un efecto significativo $(\mathrm{P}<0,01)$ en el PN .

Efecto de grupo racial. Los resultados obtenidos para los efectos de GRT y GRV sobre PN, PD205 y GDP se observan en el cuadro 4. El efecto de GRT fue significativo $(\mathrm{P}<0,001)$ para las tres variables en estudio. El GRT Angus fue $19,2 \mathrm{~kg}$ más liviano $(\mathrm{P}<0,01)$ que el GRT Hereford para PD205. El valor promedio del GRT Angus para PD205 es similar a los reportes descritos por Wilson y col (1986); Winder y col (1990); Bullock y col (1993) y Tosh y col (1999), con rangos que varían desde los 172 $\mathrm{kg}$ (Veseth y col 1993) hasta los 260,3 kg (Kaps y col 1999). Para el PN del GRT, el peso observado fue menor en la raza Angus (cuadro 4), mostrando diferencias con la raza Hereford $(\mathrm{P}<0,05)$, coincidiendo con lo reportado por Lunstra y Cundiff (2003), quienes también en-

Cuadro 4. Medias de mínimos cuadrados para peso al nacimiento (PN), peso al destete ajustado a 205 días (PD205) y ganancia de peso diaria al destete (GDP), ajustado para grupo racial del toro y de la vaca.

Least squares means for birth weight (PN), adjusted weaning weight (PD205) and pre-weaning daily gain (GDP) adjusted for breed group of the sires and dam.

\begin{tabular}{|c|c|c|c|c|c|c|c|c|c|}
\hline & & PN (kg & & & D205 $(\mathrm{k}$ & & & iDP (kg & \\
\hline & $\mathrm{N}$ & $\bar{X}$ & $\overline{\mathrm{EE}}$ & $\mathrm{N}$ & $\bar{X}$ & $\overline{\mathrm{EE}}$ & $\mathrm{N}$ & $\bar{X}$ & $\mathrm{EE}$ \\
\hline Raza de to & & & & & & & & & \\
\hline $\begin{array}{l}\text { Angus } \\
\text { Hereford }\end{array}$ & 400 & $37,0^{\mathrm{a}}$ & 0,43 & 260 & $221,6^{\mathrm{a}}$ & 4,55 & 260 & $0,89^{\mathrm{a}}$ & 0,21 \\
\hline Polled & 167 & $40,7^{b}$ & 0,53 & 127 & $240,8^{b}$ & 5,24 & 127 & $0,97^{\mathrm{b}}$ & 0,25 \\
\hline Hereford & 552 & $38,9^{\mathrm{a}}$ & 0,44 & 273 & $230,8^{c}$ & 4,75 & 273 & $0,94^{\mathrm{c}}$ & 0,22 \\
\hline Raza de la & vaca & & & & & & & & \\
\hline Clavel & 62 & $40,2^{\mathrm{a}}$ & 0,71 & 28 & $235,2^{\mathrm{a}}$ & 7,79 & 28 & $0,96^{\mathrm{a}}$ & 0,37 \\
\hline Hereford & 233 & $38,9^{\mathrm{a}}$ & 0,43 & 124 & $226,7^{\mathrm{a}}$ & 4,54 & 124 & $0,91^{\mathrm{a}}$ & 0,21 \\
\hline Híbridas & 457 & $38,8^{\mathrm{a}}$ & 0,67 & 303 & $227,7^{\mathrm{a}}$ & 6,65 & 303 & $0,92^{\mathrm{a}}$ & 0,31 \\
\hline Holstein & & & & & & & & & \\
\hline Polled & 80 & $38,6^{\mathrm{a}}$ & 0,64 & 37 & $242,4^{\mathrm{a}}$ & 6,89 & 37 & $0,99^{\mathrm{a}}$ & 0,32 \\
\hline Hereford & 287 & $38,1^{\mathrm{a}}$ & 0,43 & 168 & $223,5^{\mathrm{a}}$ & 4,63 & 168 & $0,90^{\mathrm{a}}$ & 0,22 \\
\hline
\end{tabular}

N=Número de observaciones. $\bar{X}=$ media. EE=Error estándar. a, b y c son diferentes estadísticamente $(\mathrm{P}<0,05)$. contraron diferencias significativas entre los pesos de los terneros de toros de las razas Angus y Hereford.

El efecto de GRV no fue significativo para las variables en estudio $(\mathrm{P}>0,05)$. Aún así, en el GRV Clavel se observó un mayor valor para $\mathrm{PN}$, mientras que para la variable PD205 el GRV Holstein fue superior a las otras. Dentro de la composición racial se pueden encontrar diferencias en PN desde 25,9 kg (Martínez y col 1998) hasta los 44,4 kg (Lunstra y Cundiff 2003), con una moda cercana a lo encontrado en el presente estudio (Bullock y col 1993, Tosh y col 1999).

Este amplio rango entre lo reportado para las variables PN y PD205 y lo estimado en este estudio es debido principalmente a las grandes diferencias en los componentes ambientales como la disponibilidad forrajera, condiciones agroclimáticas y diferencias en el manejo, así como en el componente materno como los distintos grupos raciales y sus cruzas (Martínez y col 1998).

Indice de herencia. Los $\mathrm{h}^{2}$ (cuadro 5) fueron estimados en forma general y dentro del GRT, con el fin de determinar si las razas paternas producían cambios en los índices de las tres variables en estudio. Los $h^{2}$ de PN, PD205 y GDP en la masa ganadera fueron de 0,49 $\pm 0,002$; $0,15 \pm 0,001$ y $0,35 \pm 0,005$, respectivamente.

Dentro de GRT se estimó el $\mathrm{h}^{2}$, encontrándose valo-

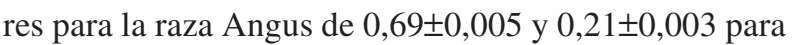

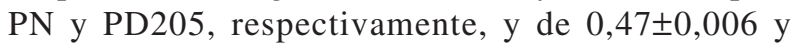
$0,23 \pm 0,001$ para la raza Polled Hereford, respectivamente. Wilson y col (1986) encontraron valores de $\mathrm{h}^{2}$ para $\mathrm{PN}$ y PD205 de 0,41 y 0,13 para la raza Hereford y de 0,19 y 0,16 para la raza Angus, respectivamente. Valores por debajo a lo reportado por Kaps y col (1999), quienes estimaron valores de $\mathrm{h}^{2}$ para PD205 en el rango de 0,53 a

Cuadro 5. Componentes de varianza, índice de herencia $\left(h^{2}\right)$, correlaciones genéticas $\left(r_{G}\right)$ y fenotípicas $\left(r_{F}\right)$ para las variables peso al nacimiento (PN), peso al destete ajustado a 205 días (PD205) y ganancia de peso diaria al destete (GDP).

Variance components, heritability $\left(h^{2}\right)$, phenotypic $\left(r_{F}\right)$ and genetic $\left(r_{G}\right)$ correlation coefficients for birth weight (PN), adjusted weaning weight (PD205) and pre-weaning daily gain (GDP).

\begin{tabular}{|c|c|c|c|c|c|c|c|}
\hline & $\begin{array}{l}\text { Primera } \\
\text { Variable }\end{array}$ & $\begin{array}{l}\text { Segunda } \\
\text { Variable }\end{array}$ & $\mathrm{Vg}$ & $\mathrm{Ve}$ & $h^{2}$ & $\mathrm{COVg}_{\mathrm{G}}$ & $r_{F}$ \\
\hline \multirow[t]{3}{*}{ General } & PN & & 13,9 & 14,4 & 0,49 & & \\
\hline & & PD205 & 384,0 & 2174,3 & 0,15 & $17,2 \quad 0,23$ & 0,22 \\
\hline & & GDP & 102,3 & 191,3 & 0,35 & $3,1 \quad 0,08$ & 0,09 \\
\hline \multirow[t]{2}{*}{ Angus } & PN & & 19,8 & 8,8 & 0,69 & & \\
\hline & & PD205 & 263,4 & 19,2 & 0,21 & $19,8 \quad 0,20$ & 0,17 \\
\hline Polled & $\mathrm{PN}$ & & 13,1 & 14,8 & 0,47 & & \\
\hline Hereford & & PD205 & 735,4 & 2528,1 & 0,23 & $47,5 \quad 0,48$ & 0,21 \\
\hline
\end{tabular}


0,59. Winder y col (1990) reportan para la raza Angus rojo estimaciones de $\mathrm{h}^{2}$ para PN y PD205 de 0,46 y 0,39, respectivamente, valores muy semejantes a los encontrados por Tosh y col (1999), quienes estimaron $h^{2}$ de 0,51 y 0,33 para PN y PD205, respectivamente, para un compuesto de 7/16 British, 1/4 Charoláis, 1/4 Simmental y 1/16 Limousine. MacNeil (2003) reporta un $\mathrm{h}^{2}$ de 0,49 y 0,30 para PN y PD, respectivamente, para la cruza de 1/2 Angus rojo, 1/4 Charoláis y 1/4 Tarentaise. Elzo y Wakeman (1998) en un trabajo con las razas Angus, Brahman y sus cruzas reportan para la raza Angus valores de 0,22 y 0,25 para $\mathrm{PN}$ y $\mathrm{PD}$, respectivamente; para la cruza $3 / 4$ Angus y $1 / 4$ Brahaman de 0,16 y 0,18 , respectivamente y para la cruza $5 / 8$ Angus $3 / 8$ Brahman de 0,13 y 0,15 para PN y PD, respectivamente. Para GPD, Veseth y col (1993) reportan un $\mathrm{h}^{2}$ de 0,20 , mientras que Winder y col (1990) lo estimaron en 0,33.

Correlación. En cuanto a las correlaciones encontradas en este trabajo, la correlación genética entre PN y PD205 tiene un valor intermedio-alto $(0,23)$, aunque este valor es inferior a los encontrados en la literatura, los cuales están entre 0,60 y 0,73 (Veseth y col 1993, Meyer 1995, Tosh y col 1999). Koots y col (1994), tras una revisión bibliográfica de 51 años reportan una estimación ponderada para la correlación entre el peso al nacimiento y peso al destete de 0,36 .

La correlación fenotípica es de 0,22, que es similar a la reportada por Veseth y col (1993), aunque Tosh y col (1999) y Meyer (1995) presenta valores superiores a los descritos, alcanzando los 0,46. La correlación genética entre PN y GPD es baja $(0,085)$, aunque intermedia entre lo reportado por otros autores como Winder y col $(1990,-0,68)$ y Veseth y col $(1993 ; 0,20)$. La correlación fenotípica alcanza un valor similar a la correlación genética $(0,088)$, donde Winder y col (1990) siguen su mismo patrón $(-0,67)$ y Veseth y col (1993) reportan un valor similar al estimado en este trabajo $(0,05)$.

En cuanto a la correlación entre PD205 y GPD, ambas (la genética y fenotípica) alcanzan una alta correlación entre ellas (1,00 y 0,999, respectivamente), siendo estos valores muy cercanos a los estimados por Veseth y col (1993; 0,95 y 0,97, respectivamente).

Los resultados del estudio permiten concluir que los valores de $\operatorname{los} \mathrm{h}^{2}$ son lo suficientemente altos para considerar programas de mejoramiento genético que permitan optimizar los parámetros productivos de peso al nacimiento y destete al considerarse los grupos raciales de toro y vaca en las condiciones ambientales de este estudio.
La raza Hereford, del grupo racial del toro, presentó la mayor eficiencia productiva y al combinarse con la raza Holstein, del grupo racial de la vaca, se observaron los menores pesos al nacimiento y los mayores pesos al destete, lo cual es un indicador de la habilidad combinatoria de estas dos razas.

La raza Polled Hereford, del grupo racial del toro, tuvo su mejor habilidad combinatoria con la raza Clavel, del grupo racial de la vaca para peso al destete y ganancia de peso diaria; sin embargo, se observó un mayor peso del ternero al nacimiento, lo cual tiene que ser considerado en las prácticas de manejo al parto.

Los resultados obtenidos tienen la importancia de describir el desempeño productivo de una masa ganadera dentro de una zona agroclimática definida $\mathrm{y}$, además, pueden servir como base para futuros análisis de tipo regional o nacional que permitan conocer las características genéticas de la ganadería chilena. De igual forma, permiten incentivar el desarrollo de mejores programas de mejoramiento animal, con el fin de alcanzar una mejor utilización de los recursos disponibles, bajando los costos y aumentando tanto la calidad como rentabilidad del sector.

\section{RESUMEN}

El objetivo del presente estudio fue determinar el comportamiento productivo al nacimiento y destete de los terneros hijos de toros Angus, Hereford y Polled Hereford con vacas Clavel, Hereford, Holstein, Polled Hereford e Híbridas (cruzas de Holstein con combinaciones de Hereford) en un rebaño bovino de carne en la provincia de Ñuble, VIII Región de Chile. Los animales fueron clasificados de acuerdo a sus características fenotípicas. El comportamiento productivo se evaluó analizando 1.119 registros productivos, desde 1999 al 2000, para las variables peso al nacimiento ( $\mathrm{PN}, \mathrm{N}=1.119$ ), peso al destete ajustado (PD205, N=660) y ganancia predestete (GDP, $\mathrm{N}=660$ ) utilizando el método de mínimos cuadrados para determinar el efecto de la raza del toro (GRT), la raza de la vaca (GRV), el grupo contemporáneo (GC) y edad de la vaca (EDV) como covariable sobre las variables PN, PD205 y GDP. Los parámetros genéticos y ambientales fueron estimados usando un modelo animal con la metodología MTDFREML, empleándose análisis univariados y bivariados para la estimación de los componentes de varianza. El efecto de GRT y GC fue significativo $(\mathrm{P}<0,05)$ para $\mathrm{PN}$ y $\mathrm{PD} 205$ pero no para GDP $(\mathrm{P} \bullet 0,05)$. La raza Hereford estuvo $7,5,12,5$ y $14,0 \%$ encima de la media del hato para PN, PD205 y GDP, respectivamente. El GRV no afectó $(\mathrm{P} \bullet 0,05)$ a las variables PN, PD205 y GDP. El índice de herencia $\left(\mathrm{h}^{2}\right)$ estimado fue de 0,49 para el PN, de 0,15 para el PDA205 y de 0,35 para el GDP. La correlación genética entre PN y PDA205 fue de 0,23 . Dentro de cada raza paterna, el $\mathrm{h}^{2}$ para PN y PD205 fueron 0,69 y 0,21 para la raza Angus y de 0,47 y 0,23 para la raza Polled Hereford, respectivamente. 


\section{REFERENCIAS}

Alejo D, M Campero, C Faverín, I Fernández. 2000. Caracterización de partos y mortalidad perinatal asociado a genotipos en ganado de carne. Veterinaria argentina 17 , 333-340.

Boldman KG, LA Kriese, LD Van Vleck, CP Van Tassel and SD Kachman. 1995. A manual for use of mtdfreml. A set of programs to obtain estimates of variances and covariances. U.S. Department of Agriculture, Agricultural Research Service.

Bullock KD, JK Bertrand, and LL Benyshek. 1993. Genetic and environmental parameters for mature weight and other growth measures in polled hereford cattle. J Anim Sci 71, 1737-1741.

Del Pozo A, P del Canto. 1999. Áreas agroclimáticas y sistemas productivos en la VII y VIII regiones. INIA. Chillán, Chile.

Dickerson G E. 1978. Animal size and efficiency: basic concepts. Anim Prod 24, 367.

Elzo MA, and DL Wakeman. 1998. Covariance components and prediction for additive and nonadditive preweaning growth genetic effects in an angus-brahman multibreed herd. J Anim Sci 76, 1290-1302.

Hatcher L and EJ Stepanski. 1997. A step-by-step approach to using the SAS system for univariate and multivariate statistics, Cary, NC, SAS Institute inc.

Kaps M, W O Herring, and E R Lamberson. 1999 Genetic and environmental parameters for mature weight in angus cattle. J Anim Sci 77, 569-574.

Koots KR, JP Gibson and JW Wilton. 1994. Analyses of published genetic parameter estimates for beef production traits. 2. Phenotypic and genetic correlations. Anim breeding abstr 62, 825-853.

Lunstra DD, and LV Cundiff. 2003. Growth and pubertal development in brahman-, boran-, tuli-, belgian blue-, hereford- and angus-sired fl bulls. J Anim Sci 81, 14141426.

MacNeil MD. 2003. Genetic evaluation of an index of birth weight to improve efficiency of beef production. J Anim Sci 81, 2425-2433.

Magofke JC. 1991. Caracterización de algunas razas bovinas de carne. I. Sobrevivencia y pesos vivos nacimientos-destete. Av Prod Anim 16, 3-20.

Martínez GJC. 1986. Mortalidad de hembras, desde el nacimiento a primer parto en bovinos de diferentes genotipos lecheros, bajo condiciones de Turrialba, Costa Rica. Tesis Maestría. Catie, Turrialba, Costa Rica. P. 132.

Martínez-González JC, FJ García-Esquivel, A González-Reyna, y A Tewolde-Medhin. 2003. Peso al nacimiento de Bos taurus, Bos indicus y sus cruces en Aldama, Tamaulipas, México. Memoria. XXXI reunión anual de AMPA. Universidad Autónoma de Chihuahua. Phoenix, Arizona. P. 217224.

Martínez G, J Patrocinio, y P Herrera. 1998. Factores que afectan el peso al nacer en un rebaño de bovinos de carne en condiciones de sabana bien drenadas. Rev Fac Agrom 15, 446-454.

Meyer K 1995. Estimates of genetic parameters and breeding values for New Zealand and Australian Angus cattle. Aust J Agric Res 46, 1219-1229.

Ruiz I. 1996. Praderas para Chile. INIA. Santiago. Chile.

Spide PL, MF Rothschild, and WW Wundor. 1981. Applied genetic. Animal Science Department of Iowa State University. U. S. A.

Simm G. 1998. Genetic improvement of cattle and sheep. $\left(1^{\text {st }}\right.$ ed.). Biddlest Ltd, Guildford and King's Lynn. Great Britain.

Swiger LA, WR Harvey, DO Everson, KE Gregory. 1964. The variance of intra-class correlation involving groups with one observation. Biometrics 20, 818-826.

Tosh JJ, RA Kemp, and DR Ward. 1999. Estimates of direct and maternal genetic parameters for weight traits and backfat thickness in a multibreed population of beef cattle. Can J Anim Sci 79, 433-439.

Varona L, I Misztal, and JK Bertrand. 1999. Threshold-linear versus linear-linear analysis of birth weight and calving ease using an animal model. I. Variance component estimation. J Anim Sci 77, 1994-2002.

Veseth DA, WL Reynolds, JJ Urick, TC Nelsen, RE Short, and DD Kress. 1993. Paternal half-sib heritabilities and genetic, environmental, and phenotypic correlation estimates from randomly selected hereford cattle. $J$ Anim Sci 71, 1730-1736.

Wilson DE, PJ Berger, and RL Willham. 1986. Estimates of beef growth trait variances and heritabilities determined from field records. J Anim Sci 63, 386-394.

Winder JA, JS Brinks, RM Bourdon, and BL Golden. 1990. Genetic analysis of absolute growth measurements, relative growth rate and restricted selection indices in red angus cattle. J Anim Sci 68, 330-336. 